\title{
Modélisation hydro-sédimentaire de la Baie du Mont Saint-Michel
}

\author{
Florence Cayocca ${ }^{1}$, Morgan Dussauze ${ }^{2}$, Pierre Le Hir $^{1}$, \\ Philippe Bassoullet ${ }^{1}$, Hervé Jestin ${ }^{3}$ \\ Ifremer, B.P.70, 29280 Plouzané, France \\ ${ }^{1}$ chercheur \\ 2 élève ingénieur \\ ${ }^{3}$ ingénieur
}

\section{Résumé}

Un modèle numérique hydro-sédimentaire de la Baie du Mont Saint-Michel a été développé pour l'étude des transferts de sédiments en relation avec les préoccupations écologiques du site. De récentes mesures de courant sur l'estran montrent l'influence du vent sur les courants de morte eau, et sont comparées aux résultats hydrodynamiques. La modélisation du transport des sédiments fins sous l'action de la marée et des vagues reproduit correctement leur répartition dans la Baie.

\section{$\underline{\text { Abstract }}$}

A numerical model for hydrodynamics and sediment transport is applied to Mont Saint Michel Bay, where ecological concerns are related to sediment transfers. Recent current datasets recorded on the tidal flat show the wind influence on the currents during neap tide. They are compared to model outputs. The simulation of sediment transfers driven by tides and waves accurately mimics the sediment repartition in the Bay.

\section{$\underline{\text { Mots-clés }}$}

Mont Saint Michel, courant, modèle, sédiments cohésifs, turbidité

\section{Introduction}

La baie du Mont Saint-Michel est l'une des principales régions de production conchylicole de la façade de la Manche. La diminution de la productivité des installations ostréicoles et mytilicoles a motivé un programme de recherche s'intéressant particulièrement à la capacité trophique de la baie (chantier PNEC Baie du Mont Saint-Michel). L'étude de l'environnement vivant est intimement liée à celle de l'environnement physique (hydrodynamique et sédimentaire) au travers de divers paramètres que sont la turbidité de l'eau (e.g. influence sur la production primaire ou sur l'écophysiologie des coquillages), la remise en suspension de sédiments (e.g. influence sur le recrutement ou la remise en suspension de microphytobenthos), l'évolution de la nature du fond (e.g. 
envasement d'installations ostréicoles). De nombreuses campagnes de mesures ont permis de cartographier la couverture sédimentaire de l'estran, et d'étudier ses évolutions (phases d'érosion et de dépôt) en fonction des conditions météorologiques et de la situation le long de l'estran ${ }^{[1]}$. La modélisation numérique des processus responsables de ces évolutions spatio-temporelles facilite la compréhension globale des phénomènes.

Dans ce contexte, un modèle numérique a été implanté sur le domaine de la Baie du Mont Saint-Michel afin d'analyser l'influence de divers paramètres sur les processus sédimentaires observés. Après une brève description du modèle, cet article analyse quelques résultats concernant le régime hydrodynamique, en particulier l'influence du vent sur les courants. Ces résultats sont confrontés à des mesures récentes. Quelques résultats du modèle sédimentaire proposé sont ensuite discutés.

\section{Description du modèle hydrodynamique}

Le modèle hydro-sédimentaire $\mathrm{SiAM} \mathrm{D}^{[2]}$ est ici utilisé dans sa version bidimensionnelle (coordonnées sigma, une seule couche). Le marnage exceptionnel de la Baie (jusqu'à $13 \mathrm{~m}$ en vive eau) est responsable d'un intense mélange sur la verticale qui justifie ce choix. La bathymétrie a été construite à partir des sources les plus récentes disponibles sur la zone :

- un levé LIDAR de 2003, dont la résolution horizontale est de $1 \mathrm{~m}$;

- $\quad$ un levé IFREMER de 2003, effectué par sondeur mono-faisceau entre $-4 \mathrm{~m}$ et $-22 \mathrm{~m}$ (IGN69, profils espacés de $200 \mathrm{~m}$ ) ;

- les cartes du SHOM pour les zones non couvertes par les levés ci-dessus.

Le traitement de ces données a été effectué à l'aide du logiciel de géostatistiques Isatis (http://www.geovariances.fr/), qui a permis de vérifier en particulier le bon accord entre le levé traditionnel par bateau (IFREMER) et le levé LIDAR. Les comparaisons entre jeux de données ont été effectuées après avoir construit des modèles numériques de terrain (MNT) au pas de $100 \mathrm{~m}$. A l'exception de la zone sableuse des chenaux, où la très forte dynamique de la morphologie peut expliquer les différences observées entre les deux sources d'information (les acquisitions ont eu lieu à plusieurs mois d'intervalle), la moyenne et l'écart-type des différences entre ces deux sources sur leur zone de recouvrement sont respectivement de $2 \mathrm{~cm}$ et $26 \mathrm{~cm}$ (la précision des deux systèmes est par ailleurs estimée à une vingtaine de centimètres, J. L'Yavanck et J. Populus, comm. pers.). Pour comparaison, à la limite entre les données IFREMER 2003 et les données des cartes SHOM, la moyenne et l'écart type des différences sont respectivement de $14 \mathrm{~cm}$ et $91 \mathrm{~cm}$. Les données du SHOM, plus anciennes, concernent néanmoins des zones de grande hauteur d'eau et de faible gradient bathymétrique, où les erreurs sur la bathymétrie ont un effet modéré sur les résultats de simulation des courants.

La résolution spatiale du maillage (cartésien irrégulier) varie de 200 à $500 \mathrm{~m}$ (Figure 1). 
Les conditions aux limites du large du modèle sont fournies en hauteur d'eau, et sont calculées à partir de 115 harmoniques de marée ${ }^{[3]}$. Les débits des 3 fleuves principaux (Couesnon, Sée, Sélune) sont prescrits. Le vent est considéré uniforme sur le domaine de simulation, et provient d'un point de sortie du modèle Aladin (Météo France) situé au milieu de la Baie du Mont Saint Michel. L'écart de norme entre le vent résultant de l'analyse Aladin et le vent mesuré à la station météorologique de Dinard (située à $16 \mathrm{~km}$ à l'Ouest de Cancale) est de l'ordre de $20 \%$, et est indépendant de la direction. Les variations en direction entre les sorties du modèle et les mesures à Dinard sont comprises entre $-45^{\circ}$ et $45^{\circ}$. On considère dès lors qu'il est légitime d'utiliser le vent modélisé, sachant que les séries temporelles mesurées sont souvent incomplètes.

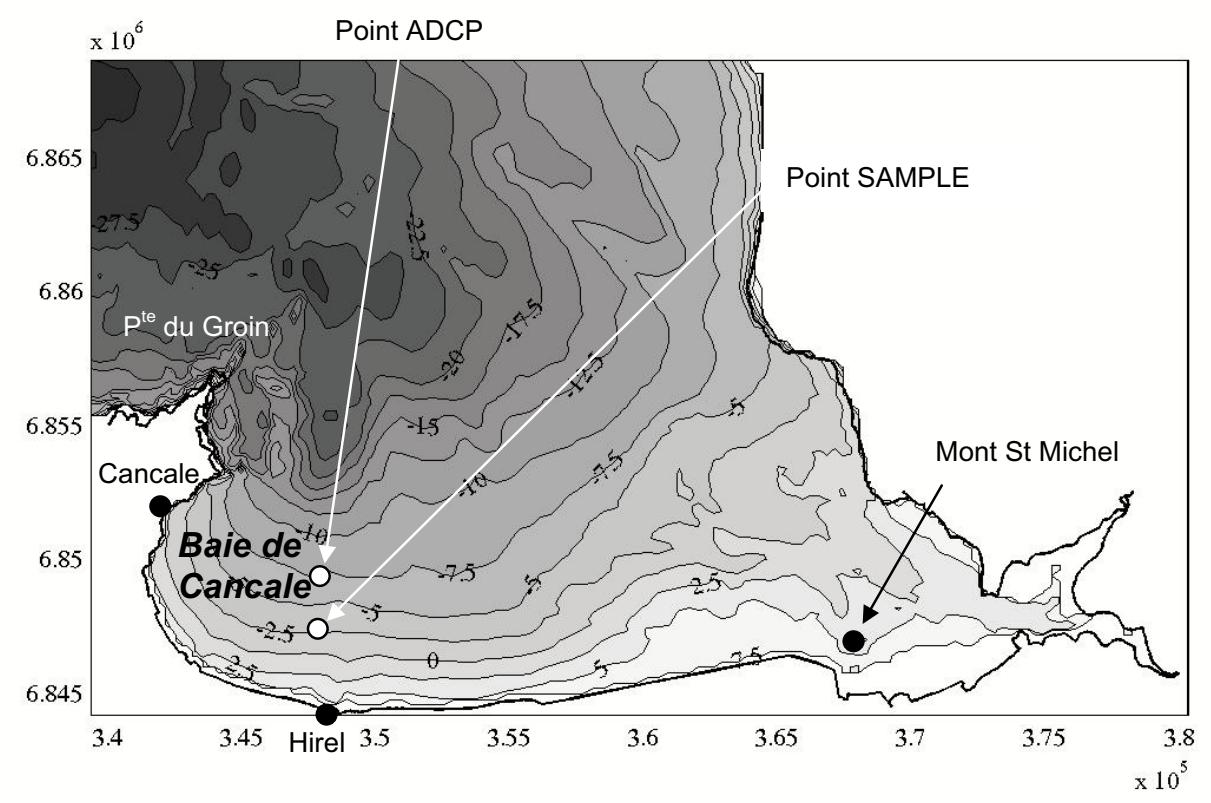

Figure 1 : Bathymétrie. Les coordonnées sont données dans la projection Lambert 93, et les hauteurs d'eau se réfèrent au niveau moyen.

\section{Résultats et mesures}

Plusieurs modèles hydrodynamiques ont décrit les régimes de courants en Baie du Mont Saint Michel ${ }^{[4]}$. Les caractéristiques principales concernent les fortes vitesses rencontrées devant la pointe du Groin et dans les chenaux du Sud-Est de la Baie. Vis-à-vis de la dynamique sédimentaire, la représentation des contraintes sur le fond maximales (Figure 2) permet de constater une bonne corrélation entre hydrodynamisme de marée et couverture sédimentaire : on observe non seulement un gradient "cross-shore » des contraintes sur le fond, mais aussi un gradient " longshore ", avec des contraintes maximales plus faibles à l'Ouest de la Baie que dans la partie orientale. Les courants de marée peuvent donc, du moins en partie, expliquer l'affinement des sédiments vers l'ouest. 


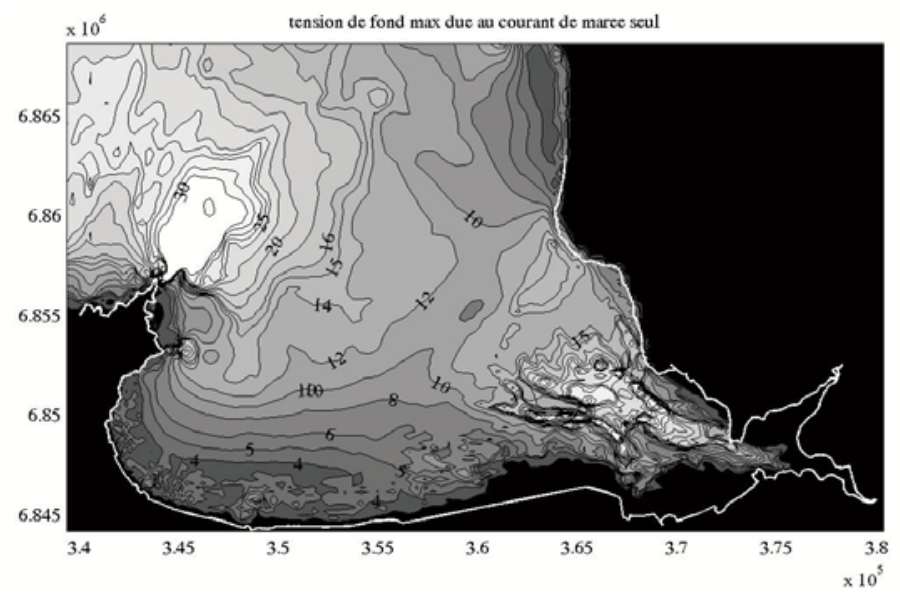

Figure 2 : Tension sur le fond maximales calculées en vive eau (N.m $\left.{ }^{-2}\right)$

Les mesures de courant jusqu'alors disponibles ont été répertoriées par BonnotCourtois $^{[5]}$; elles ont pour la plupart été effectuées sur un seul cycle de marée, et aucune ne concerne l'estran. De nouvelles mesures de long terme ont été effectuées en 2003, 2004 et 2005. Nous utilisons ici deux séries, l'une effectuée par courantomètre Doppler posé sur le fond (ADCP RDI Sentinel 1200kHz, point ADCP figure 1) en mars 2005, et l'autre par courantomètre électromagnétique à $30 \mathrm{~cm}$ du fond sur la station SAMPLE en mars 2003. La comparaison des mesures avec le modèle se fait par intégration sur la verticale dans le cas des mesures en profil, et en supposant un profil logarithmique des vitesses pour la mesure SAMPLE.

Les mesures sur estran montrent une très forte variabilité en morte eau (Figure 3), qui peut en partie être expliquée par le vent. En particulier, le vent d'Est induit une composante zonale des vitesses nettement dirigée vers l'Ouest (13 et 14 mars), tandis qu'elle porte en moindre mesure vers l'Est par vent d'Ouest (10 et 11 mars). L'effet du vent de Nord ou de Sud est moins clair. On peut par exemple observer la composante méridienne du courant dirigée vers le Nord tout au long de la marée par vent de Nord (exemple le 12 mars). Le modèle représente bien cette forte variabilité, et les séries temporelles (non montrées ici) montrent que l'amplitude des vitesses est bien simulée. En vive eau, le vent n'a plus d'influence sur les vitesses. La direction du courant ne présente pas la forte variabilité observée en morte eau, et les courants sont alternatifs (Figure 4).

Plus au large (point ADCP), le courant moyenné sur la verticale est indépendant $\mathrm{du}$ vent. L'amplitude du courant varie de $10 \mathrm{~cm} \cdot \mathrm{s}^{-1}$ à $80 \mathrm{~cm} \cdot \mathrm{s}^{-1}$, avec une dominance du flot qui augmente avec l'amplitude de la marée (vitesses de flot supérieures de 25 à $30 \%$ aux vitesses de jusant en vive eau). L'allure des roses de courant est invariante sur toute la durée de la mesure (courant rotatif, figure 5). Le modèle reproduit correctement les amplitudes; en revanche, les courants prédits sont plutôt alternatifs. 


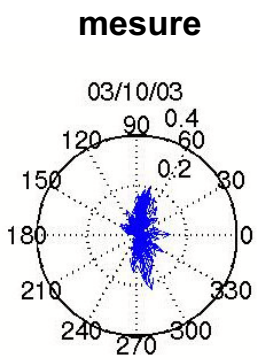

\section{modèle}

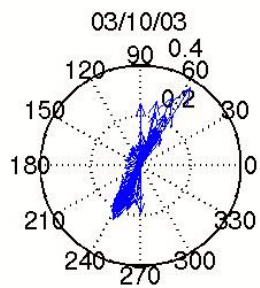

$03 / 11 / 03$

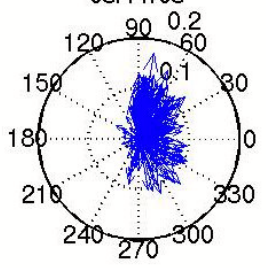

$03 / 12 / 03$

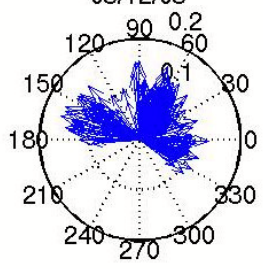

$03 / 11 / 03$

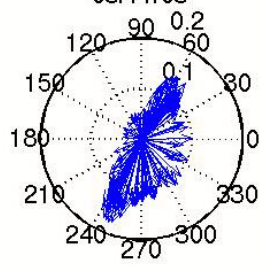

$03 / 12 / 03$

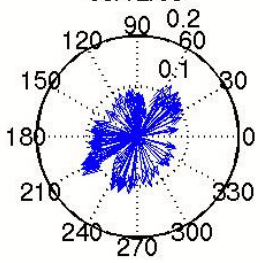

mesure

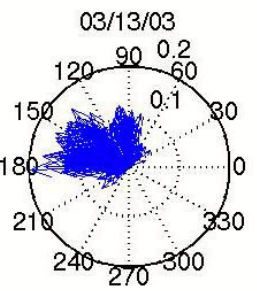

$03 / 14 / 03$

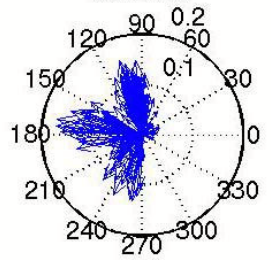

$03 / 15 / 03$

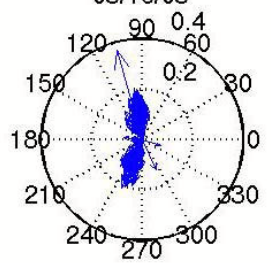

modèle
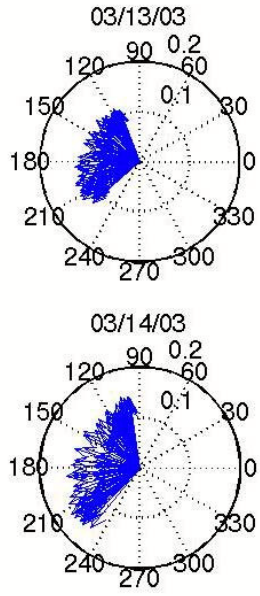

$03 / 15 / 03$

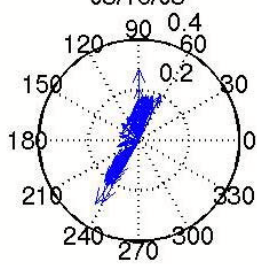

Figure 3 : Courants mesurés par la station Sample en morte eau (du 10 au 15 mars 2003, colonnes 1 et 3 ) et courant simulé par le modèle aux mêmes dates (colonnes 2 et 4). Vent de Sud-Ouest les 10 et 11 mars, virant de l'ouest à l'est par le nord le 12 , et vent d'est du 13 au 15 ; intensités de 7 à $10 \mathrm{~m} \cdot \mathrm{s}^{-1}$.

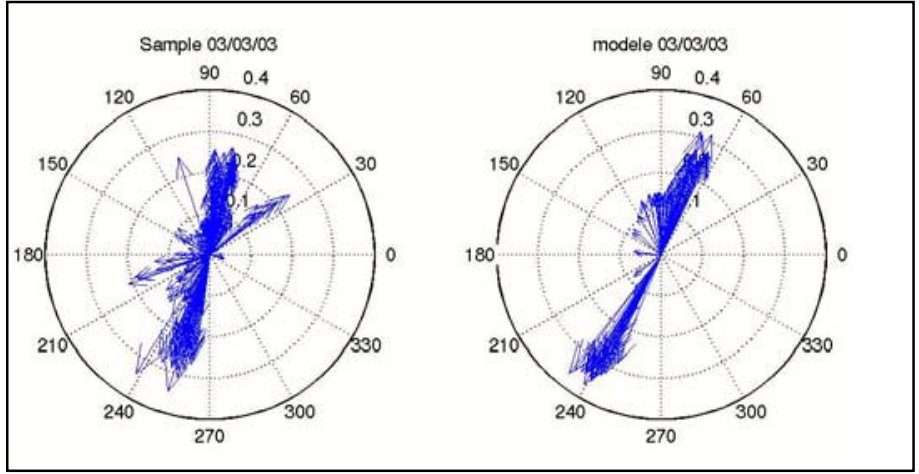

Figure 4 : Courant mesuré par Sample (point SAMPLE, à gauche) et simulé par le modèle (à droite), le 3 mars 2003 (coefficient 91).

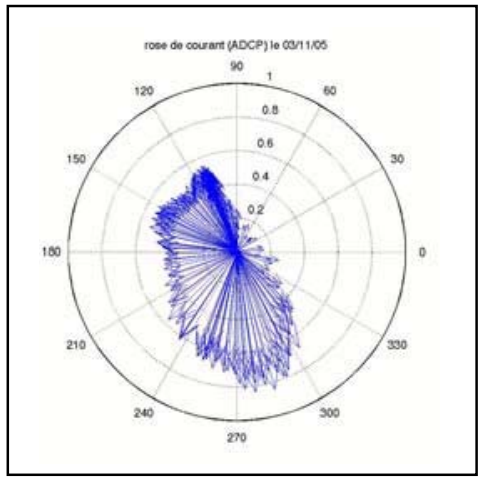

Figure 5: Exemple de mesure par ADCP (au point ADCP) le 11 mars 2005 (coefficient 110) 


\section{Modélisation des dépôts de vase et turbidité}

Une particularité de la Baie du Mont Saint Michel est la grande variabilité de la couverture sédimentaire dans la direction Est-Ouest (du Mont Saint Michel très sableux à l'Est à la Baie de Cancale principalement vaseuse à l'Ouest) et dans la direction " cross-shore », avec un haut estran très vaseux au-dessus d'anciennes pêcheries qui jouent aujourd'hui le rôle de pièges à sédiments ${ }^{[6]}$.

Dans cet environnement complexe, une première approche consiste à ne considérer qu'une fraction cohésive dont on simule au mieux le comportement. On fait alors l'hypothèse implicite que la répartition de cette fraction dépend peu de la présence des fractions plus grossières (ce qui n'est qu'une approximation), et que le modèle sera valide s'il reproduit correctement la répartition de la fraction fine. Par ailleurs, la turbidité dans la Baie est essentiellement due aux sédiments fins. On peut alors considérer que le signal de turbidité simulé par le modèle doit être conforme aux niveaux de turbidité mesurés.

Un calcul de fetch a été réalisé sur le domaine afin de prendre en compte les vagues. Les limitations d'une telle approche sont connues :

- les vagues générées sur le domaine ne dépendent que du vent local, ce qui ne permet pas la prise en compte d'une variation de forçage météorologique le long du parcours de la vague ;

- un corollaire de la précédente limitation est l'impossibilité de propager de la houle générée au large.

Avant la mise en place d'un modèle de propagation complet sur la zone, il est néanmoins instructif d'évaluer si l'utilisation d'un simple modèle de fetch peut fournir des résultats satisfaisants. L'approche retenue consiste à calculer, pour chaque point du maillage et pour chaque secteur angulaire de $7.5^{\circ}$, la distance à la côte la plus proche (i.e. le fetch). La hauteur de vague en chaque point et à chaque instant dépend d'une part de l'intensité du vent durant les 3 jours précédents, d'autre part du fetch sur lequel ce vent a soufflé. Les expressions utilisées sont celles proposées par Hurdle et Stive ${ }^{[7]}$. En eau peu profonde, une limitation supplémentaire est appliquée à partir de la hauteur d'eau locale (et non de la hauteur d'eau tout au long de la propagation de la vague ; formulation SEPM, 1984). Les résultats de ces calculs ont pu être comparés à des mesures effectuées simultanément en un point du large (bouée DW4, campagne EPEL ${ }^{[8]}$ ) et en un point de mesure sur estran (station multi-paramètre SAMPLE). Cette analyse montre que le calcul de fetch fournit des hauteurs de vagues réalistes après correction par un facteur uniforme sur l'estran (où les vagues ont un effet sur la remise en suspension). Cet ajustement est requis pour corriger le fait que le calcul de fetch considère une hauteur d'eau infinie sur la trajectoire de génération des vagues, ce qui n'est pas le cas ici. L'ajustement a pour effet de sous-estimer les vagues du large. 
Les paramètres $\mathrm{du}$ modèle régissant le comportement sédimentaire sont déterminés à partir de mesures effectuées par le laboratoire et de données de la littérature. En particulier, le flux d'érosion $E$ est calculée à partir de la formule de Partheniades $\left(E=E_{0}\left(\tau-\tau_{c r} / \tau_{c r}\right)\right)$, où la contrainte critique d'érosion $\tau_{c r}$ et le flux d'érosion $E_{0}$ sont estimés à partir de tests d'érodimétrie in situ effectués sur des vases de Cancale et Hirel ${ }^{[9]}$. Les valeurs de $\tau_{c r}$ mesurées varient entre 0.25 et $3 \mathrm{~N} . \mathrm{m}^{-2}$ tandis que $E_{0}$ est de l'ordre de $0.2 \mathrm{~g} . \mathrm{m}^{-2} \cdot \mathrm{s}^{-1}$. La densité de la vase de surface varie de $530 \mathrm{~g} .1^{-1}$ (vase liquide) à $1200 \mathrm{~g} . \mathrm{l}^{-1}$ (vase silteuse indurée). Le paramétrage du tassement des vases dans le modèle est fait de manière à se conformer aux résultats de culots de tassement de Migniot ${ }^{[10]}$. Les processus d'érosion, dépôt et tassement sont représentés comme suit : un sédiment érodé est mis en suspension, puis est transporté par les courants avant de se redéposer à une concentration de vase fraîche que l'on prescrit arbitrairement. Le dépôt intervient lorsque la tension de fond est inférieure à une tension critique de dépôt. Le sédiment déposé peut alors se tasser, soit en étant préalablement mélangé au sédiment sous-jacent (dont il modifie alors la densité), soit en se tassant « indépendamment ».

Pour le résultat présenté ici, la configuration initiale suppose une couverture uniforme et homogène de $10 \mathrm{~cm}$ vase de concentration $800 \mathrm{~g} .1^{-1}$ au sud d'un parallèle passant au sud de Cancale (Figure 6). Le tassement étant pris en compte, $\tau_{c r}$ est paramétré en fonction de la concentration $C$ du sédiment de surface en $\mathrm{g} . \mathrm{l}^{-1}$, selon la formulation suivante :

$\tau_{c r}=x_{1} C^{x 2}$ où $x 1=2.10^{-7}$.et $x 2=2.2$. Les valeurs de $x 1$ et $x 2$ permettent de reproduire les résultats de Migniot, et fournissent des valeurs de contrainte compatibles avec les mesures effectuées avec l'érodimètre ${ }^{[9]}$.

Après quelques marées simulées, ces dépôts « artificiels » se réorganisent pour se restreindre à l'estran. La figure 6 doit être interprétée avec précaution : ces épaisseurs dépendent en effet de la condition initiale prescrite. En revanche, le fait que des sédiments se maintiennent ou s'épaississent dans une zone doit y traduire la présence effective de vase. C'est bien le cas sur l'estran, avec en particulier une forte tendance au dépôt au nord de la digue située juste à l'ouest du Mont Saint Michel (zone uniquement submergée en grande vive eau, Figure 6). 


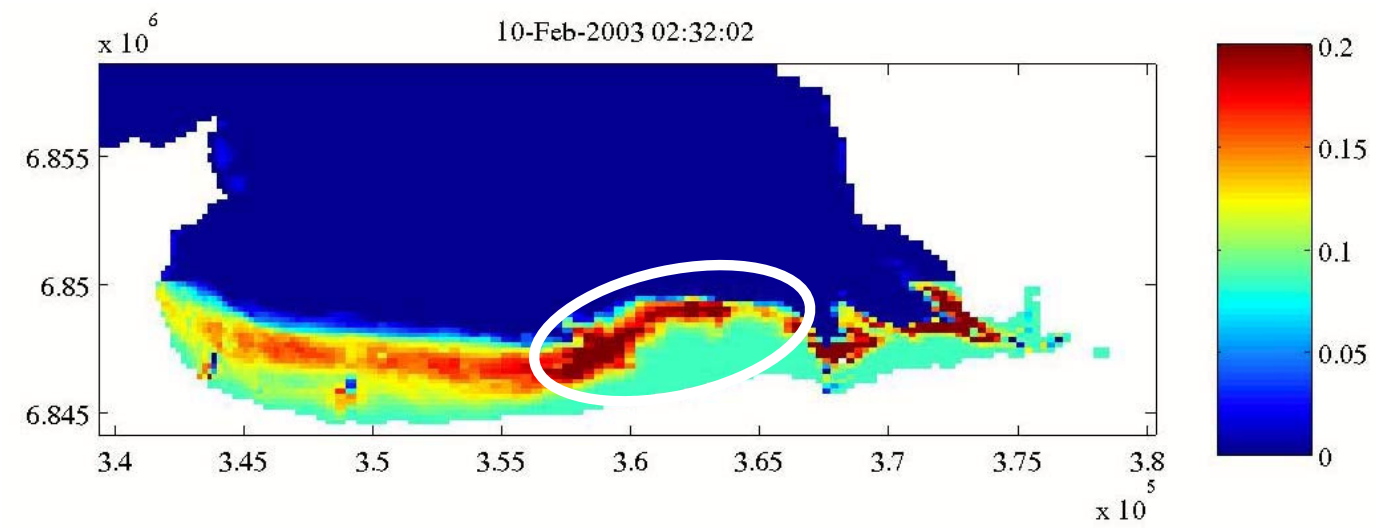

Figure 6 : épaisseur du dépôt de vase en mètres après 40 jours de simulation (l'ellipse souligne la zone de forts dépôts mentionnée dans le texte).

Le haut d'estran n'est modifié que lors des marées de vive-eau. Cela explique que les concentrations du sédiment de surface soient plus élevées en haut d'estran qu'en milieu d'estran, puisqu'il n'y a pas d'apport de dépôts frais en haut d'estran durant toute la morte eau (Figure 7). Le sédiment peut donc davantage s'y compacter. En revanche, le modèle ne prédit pas une tendance particulière à l'envasement à Cancale. Deux hypothèses peuvent expliquer ce résultat : d'une part, le modèle ne prend pas en compte les installations conchylicoles. D'autre part, la prise en compte des vagues est faite de manière sommaire. La simulation de la propagation des vagues à l'aide d'un modèle énergétique a montré que le gradient «longshore» des contraintes sur le fond dues à la houle est plus important que dans cette simulation ${ }^{[11]}$.

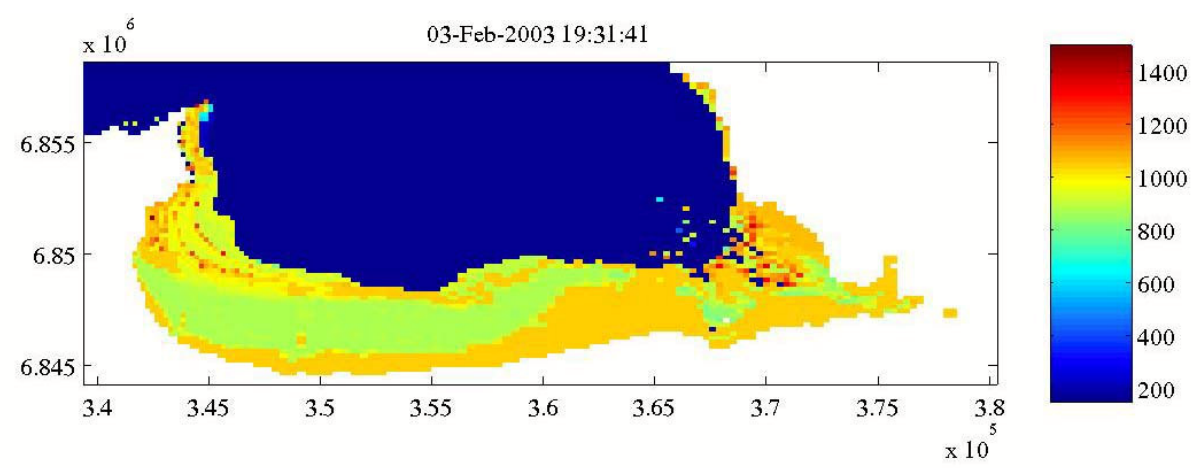

Figure 7 : Concentration du sédiment de surface (en g. $1^{-1}$ ). Les valeurs inférieures à 400 g. $1^{-1}$ concernent des zones où aucun dépôt de vase n'est simulé. Par ailleurs, des dépôts frais peuvent apparaître temporairement, et être érodés à la marée suivante.

Après l'établissement d'une situation d'équilibre où les dépôts sédimentaires sont en accord avec le forçage hydrodynamique, on peut examiner les valeurs de turbidité simulées. La figure 8 représente les valeurs moyennes et maximales de turbidité simulées de mi-janvier à mi-février 2003. Les valeurs maximales de l'ordre de $1.5 \mathrm{~g} . \mathrm{l}^{-1}$ atteintes sur toute la largeur de l'estran correspondent aux périodes de recouvrement et de découvrement, et sont du même ordre de grandeur 
que les valeurs mesurées au point fixe sur estran (de 1 à 2 g. l $^{-1}$ selon les périodes et les endroits). Le modèle prédit en moyenne des concentrations en suspension plus élevées dans la partie occidentale de la baie. Ce résultat est difficile à confirmer: les mesures de turbidité effectuées en 2003 au centre de la baie (Cherrueix, moyenne de $0.35 \mathrm{~g} . \mathrm{l}^{-1}$, communication Levoy), concernent des mélanges de vase et sable fin en suspension, et ne peuvent être comparées à des résultats de simulation de vase seule. Deux séries de mesures sur estran dans la partie occidentale de la baie (Cancale et Hirel) au printemps 2004 donnent en revanche des valeurs moyennes de $0.3 \mathrm{~g} .1^{-1}$, que le modèle représente en moyenne correctement.
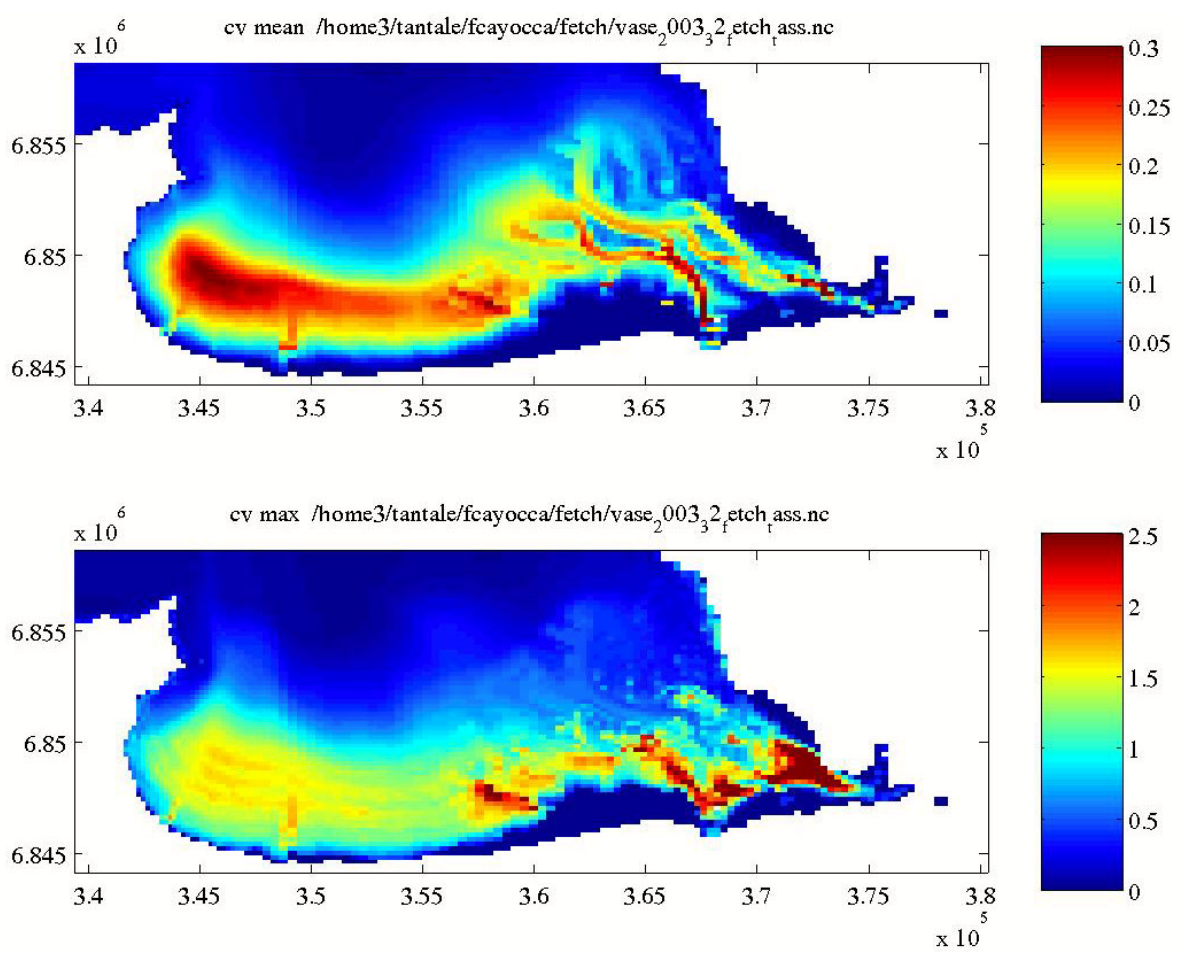

Figure 8 : Concentrations moyennes (en haut) et maximales (en bas) exprimées en g.1-1, calculée sur une période allant de mi-janvier à mi-février 2003.

\section{Conclusions}

Des mesures de courant de plusieurs semaines ont permis de montrer la grande variabilité des courants sur l'estran en morte eau due à leur forte sensibilité au vent. En vive eau sur l'estran et à toutes périodes dans le reste du domaine, les courants sont plus réguliers. Le modèle SiAM reproduit la forte variabilité sur estran, et permet de simuler des amplitudes comparables aux mesures. Il sousestime néanmoins la composante zonale du courant dans la partie occidentale de la Baie (les modèles précédemment implantés sur la zone ne fournissent pas de représentation permettant d'estimer cette propriété). 
A partir d'une couverture sédimentaire schématique, le modèle évolue pour simuler une répartition cohérente des sédiments fins, et des ordres de grandeur de turbidité conformes aux mesures.

\section{Bibliographie}

1 Bonnot Courtois, C., Bassoullet, P., Le Hir, P., Tessier, B., Cayocca, F., Baltzer, A., (2006). Evolution et mobilité des sédiments superficiels de l'estran occidental de la Baie du Mont Saint Michel. Actes de colloque GCGC2006, Brest, France 2 Le Hir, P., Cayocca, F., (2002). 3D application of the continuous modelling concept to mud slides in open seas. J.W.C. Kranenburg (Editor), Fine Sediment Dynamics in the Marine Environment. Proceedings in Marine Science No5. Elsevier, pp. 545-562.

3 Le Roy, R., Simon, B., (2003). Réalisation et validation d'un modèle de marée en Manche et dans le Golfe de Gascogne; Application à la réalisation d'un nouveau programme de réduction des sondages bathymétriques. Rapport d'études SHOM 002/03.

4 Ehrhold, A., (1999). Dynamique de comblement d'un bassin sédimentaire soumis à un régime mégatidal : Exemple de la Baie du Mont Saint Michel, thèse de l'Université de Caen, 294 pp.

5 Bonnot-Courtois, C., Caline, B., L'Homer, A., Le Vot, M. (2002). Baie du Mont Saint-Michel et Estuaire de la Rance : Environnements sédimentaires, aménagements et évolution récente.

Editions TotalFinaElf. Mémoire $\mathrm{n}^{\circ} 26,256 \mathrm{pp}$.

6 Bahé, S., (2003). Conchyliculture et dynamique morpho-sédimentaire en Baie du Mont Saint-Michel : mise en place d'une base de données géographiques. Mémoire de DEA, EPHE, Laboratoire de géomorphologie et Environnement Littoral, $161 \mathrm{pp}$.

7 Hurdle, Stive, (1989). Revision of SPM 1984 Wave Hindcast Model to avoid inconsistencies in engineering applications. Coastal Engineering, 12, 339-351.

8 Collard, F. Ardhuin, F., Chapron, B., (2005). Extraction of Coastal Ocean Wave Fields from SAR images. IEEE Journal of Oceanic Engineering, Vol 30.(3), pp. 526-533.

9 Le Hir, P., Cann, P., Waeles, B., Jestin, H., Bassoullet, P., (2005). Erodability of natural sediments : experiments on sand/mud mixtures from laboratory and field erosion tests. Proceedings INTERCOH 2005, SAGA, Japan

10 Migniot, C., (1989). Tassement et rhéologie des vases, Parties 1 et 2.

La Houille Blanche, $\mathrm{n}^{\circ} 1-1989,11-31$; n²-1989, 95-112.

11 Chini, N., (2005). Prise en compte de la houle dans un modèle hydrosédimentaire : application au transport sédimentaire dans la Baie du Mont Saint Michel. Rapport de Master 2, IFREMER. 